\title{
CLINICOPATHOLOGICAL CHARACTERISTICS OF PATIENTS WITH SYNCHRONOUS PRIMARY OVARIAN AND ENDOMETRIAL CANCERS
}

\author{
Samreen Chaudry ${ }^{1}$, Tabinda Sadaf ${ }^{1}$, Sumera Butt ${ }^{1}$, Aamir Ali Syed ${ }^{2}$, Neelam Siddiqui ${ }^{3}$ \\ ${ }^{1}$ Department of Radiation Oncology, Shaukat Khanum Memorial Cancer Hospital and Research Centre, Lahore, Pakistan, \\ ${ }^{2}$ Department of Surgical Oncology, Shaukat Khanum Memorial Cancer Hospital and Research Centre, Lahore, Pakistan, \\ ${ }^{3}$ Department of Medical Oncology, Shaukat Khanum Memorial Hospital and Research Centre, Lahore, Pakistan
}

Received: 26 February 2016 / Accepted: 20 July 2016

\begin{abstract}
Purpose: Synchronous primary endometrial and ovarian cancers are infrequent. The objective of this study is to evaluate clinicopathological characteristics of synchronous endometrial and ovarian cancers treated in our institution.

Materials and Methods: The clinicopathological characteristics of 12 patients with synchronous ovarian and endometrial cancers treated at Shaukat Khanum Memorial Cancer Hospital and Research Centre, Lahore, Pakistan, from July 2005 to July 2015, were reviewed retrospectively in depth from hospital database. The WHO committee classification was used for the histologic determination and staged based on International Federation of Gynaecology and Obstetrics (FIGO) staging.

Results: The median age at the time of diagnosis was 50 years (range 23-66 years). The incidence of synchronous primary endometrial and ovarian cancers was $2.01 \%$ in patients with endometrial cancer. A total of seven patients were menopausal (58\%) and eight patients were nulliparous (66\%); the median body mass index (BMI) was $29 \mathrm{~kg} / \mathrm{m}^{2}$ (range, 20-38). Abnormal uterine bleeding was the most common presenting symptom. According to FIGO stage, 10 cases of endometrial were Stage I/II (84\%) and two cases were Stage III (16\%). Of the ovarian cancers, nine cases were Stage I/II $(83.3 \%)$ and two cases were Stage III (16.7\%). Endometrial cancer was the main pathological type in uterine carcinoma (86\%) followed by serous carcinoma (14\%), and similarly, for ovarian cancer, endometrial was the most common pathology (67\%) followed by serous/clear cell (16\%) and mucinous (16.7\%). Most endometrial and ovarian primaries in our series were Grade I and II tumours, $83 \%$ and $66 \%$, respectively. 8 patients $(66 \%)$ had similar histology in both primaries. All patients underwent surgical intervention. Only one patient did not receive any post-operative adjuvant therapy. 10 patients received platinum-based adjuvant chemotherapy and six patients received adjuvant radiotherapy.
\end{abstract}

Conclusion: Synchronous primary endometrial and ovarian cancers are infrequent and distinct set of patients. Abnormal per vaginal bleed was the most common symptom which helped in early detection. Majority of the patients belong to concordant endometrial histology, low grade, had younger age and high BMI. Treatment should be tailored to the stage, histology and grade of the individual tumours.

Key words: Endometrial cancer, ovarian cancer, synchronous tumours

\section{Introduction}

Synchronous primary tumours of the endometrium and ovary are relatively uncommon, representing the most common combination of synchronous primary cancer of the female genital tract. ${ }^{[1-3]}$ Approximately 5\% of all

\footnotetext{
Correspondence: Dr. Samreen Chaudry, Department of

Radiation Oncology, Shaukat Khanum Memorial

Cancer Hospital and Research Centre, Lahore, Pakistan.

Email: radidoc@skm.org.pk
}

patients with endometrial cancer appear to have ovarian cancer synchronously and $10 \%$ other way around. ${ }^{[4]}$

The pathogenesis remains unclear. According to the theory of a secondary Mullerian system, epithelium of cervix, uterus, fallopian tubes, ovaries and peritoneal surface has shared molecular receptors responding to carcinogenic stimulus, leading to the development of multiple primary malignancies synchronously. ${ }^{[5,6]}$ However, no absolute specific criteria exist to determine whether they represent 
metastasis, independent primary tumours or dysplastic process involving the Mullerian epithelium.

Patients with synchronous primary endometrial and ovarian cancers have distinct clinical characteristics including young age, obesity, premenopausal status and nulliparity. ${ }^{[7]}$ The aim of the present retrospective study is to evaluate the clinicopathological characteristics of synchronous endometrial and ovarian cancer treated in our institution.

\section{Materials and Methods}

From July 2005 to July 2015, the hospital tumour registry and information systems identified 12 patients with synchronous ovarian and endometrial cancer. Electronic and archived medical records were accessed to obtain clinicopathological data including age at diagnosis, presenting symptoms, body mass index (BMI), parity, treatment, histological subtypes and stage of disease.

Of these 12 patients, eight patients presented with per vaginal bleeding and were diagnosed with primary uterine carcinoma on endometrial curetting. These patients underwent upfront total hysterectomy and bilateral salpingooophorectomy, whereas four patients underwent upfront total hysterectomy, bilateral salpingo-oophorectomy, pelvic lymphadenectomy, omentectomy and peritoneal washings for suspected ovarian primary. The surgical specimens were reviewed by our pathologists and the diagnosis of synchronous malignancies was made according to the criteria by Scully et al ${ }^{[8]}$ All patients had incidental finding of synchronous second primary on surgical specimens.

Adjuvant therapy was proposed for selected patients based on an evaluation of the risk factors for the two synchronous carcinomas such as stage, grade, histology and accuracy of surgical staging.

Patients were considered at low risk, if they had endometrial cancer Stage IA Grade 1-2 and/or ovarian cancer Stage IA-B Grade 1; at intermediate risk, if they had endometrial cancer Stage IA Grade 3 and Stage IB Grade 1-2, and/or ovarian cancer Stage IA-B Grade 2 and at high risk, if they had endometrial cancer Stage IB Grade 3, Stage IIB and clear cell or serous carcinoma, and/or ovarian cancer Stage IC or any Grade 3. The risk of Stage IIA endometrial cancers was defined according to grade and depth of myometrium invasion.
Adjuvant chemotherapy was given to patients with intermediate and high-risk ovarian cancer.

Whereas adjuvant vaginal brachytherapy was given to intermediate or high-risk Stage I endometrial carcinomas, external beam radiotherapy along with vaginal brachytherapy was given to Stage II endometrial carcinoma.

Follow-up examinations were conducted every $3-4$ months during the first 2 years, every 6 months during next 3 years and then once a year.

\section{Results}

The median age of the 12 patients was 50 years (range $23-66$ years). Four of the patients were aged $<50$, whereas eight patients were aged $>50$. The median BMI was $29 \mathrm{~kg} / \mathrm{m}^{2}$ (range 20-38), 8 (67\%) of the 12 patients were obese $(\mathrm{BMI}>28)$. A total of seven patients were menopausal (58\%) and eight patients were nulliparous (66\%). The most common presenting symptom was abnormal uterine bleeding occurring in 6 patients (50\%), abdominal pain and abdominal fullness in 5 patients (42\%) and only one case exhibited vaginal discharge [Table 1].

Of the 12 patients, eight had hysterectomy and bilateral salpingo-oophorectomy and the remaining four patients had total hysterectomy, bilateral salpingo-oophorectomy, omentectomy with appendectomy and pelvic lymph node dissection.

The histopathological characteristics of the endometrial cancers are listed in Table 2 . We found that $50 \%$ of patient had Grade I tumours and 42\% had International Federation of Gynaecology and Obstetrics (FIGO) Stage I cancers. Myometrium invasion was seen in $50 \%$ of patients and lymphovascular space invasion was not found in any patient.

The histopathological characteristics of the ovarian cancer are listed in Table 3. We found that $42 \%$ had FIGO Stage I cancers and $50 \%$ had Grade I cancers. Endometrial cancer was the main pathological type in uterine carcinoma (86\%) followed by serous carcinoma (14\%), and similarly, for ovarian cancer, endometrial was the most common pathology (67\%) followed by serous/clear cell (16\%) and mucinous (16.7\%).

Concordant endometrial/endometrial histology was seen in $68 \%(8 / 12)$, endometrial/mucinous in $16 \%(2 / 12)$, 
Table 1: Patient characteristics

\begin{tabular}{l|c}
\hline Patient characteristics & Number (\%) \\
\hline Age & \\
\hline$<50$ & $4(23)$ \\
\hline$>50$ & $8(67)$ \\
\hline Pre-menopausal & $6(50)$ \\
\hline Postmenopausal & $6(50)$ \\
\hline Comorbid conditions & \\
\hline Diabetes & $3(25)$ \\
\hline Hypertension & $4(33)$ \\
\hline Presenting symptoms & \\
\hline Abnormal bleeding & $6(50)$ \\
\hline Abdominal/pelvic mass & 0 \\
\hline Abdominal distension & $3(25)$ \\
\hline Abdominal/pelvic pain & $2(17)$ \\
\hline Others & $1(8)$ \\
\hline
\end{tabular}

Table 2: Tumour characteristics endometrial cancer

\begin{tabular}{l|c}
\hline Characteristics & Frequency number (\%) \\
\hline Stage & $5(42)$ \\
\hline IA & $4(33)$ \\
\hline IB & $2(17)$ \\
\hline II & $1(8)$ \\
\hline III & \\
\hline Myometrium invasion & $6(50)$ \\
\hline$<50 \%$ & $6(50)$ \\
\hline$>50 \%$ & \\
\hline Grade & $6(50)$ \\
\hline I & $4(33)$ \\
\hline II & $2(17)$ \\
\hline III & $10(86)$ \\
\hline Histology & $2(14)$ \\
\hline Endometrial & \\
\hline Serous & $5(42)$ \\
\hline Adjuvant radiotherapy & $1(8)$ \\
\hline Vaginal brachytherapy & $6(50)$ \\
\hline External pelvic and vaginal & \\
\hline brachytherapy & \\
\hline None & \\
\hline
\end{tabular}

endometrial/serous in $8 \%(1 / 12)$ and endometrial/ mucinous in $8 \%(1 / 12)$ of patients.

A total of 10 patients received platinum-based adjuvant chemotherapy, five patients received both chemotherapy and radiotherapy and one patient received radiotherapy alone.

\section{Discussion}

The results of our study reflect the interesting data about the biological behaviour of synchronous endometrial and ovarian carcinomas. Reported incidence rates in literature range from $2 \%$ to $10 \% ;^{[7-12]}$ however it is $2.01 \%$ of all early-stage endometrial cancers treated at our institution.

The median age at diagnosis was 50 years. The previous studies have also reported a younger median age in patients with synchronous endometrial and ovarian cancers. It is worth mentioning, 3 women ( $25 \%$ ) were under 40 years of age at the time of diagnosis. These data support previous reports about the development risk of coexisting cancer and the common presentation of synchronous cancer in premenopausal or women of reproductive age. ${ }^{[2,13,14]}$

Obesity is well-known risk factor for the development of endometrial cancer. Soliman et al. reported that 35\% of patients were obese with BMI $>30 .{ }^{[7]}$ In the study by Nishimura et al., the mean BMI of Japanese females with synchronous primary endometrial and ovarian cancers was $22.6 \pm 3.4 \mathrm{~kg} / \mathrm{m}^{2}$, but the authors did not report the obesity rates. ${ }^{[15]}$ In the present study, the median BMI was $29 \mathrm{~kg} / \mathrm{m}^{2}$ (range 20-38), 8 (67\%) of the 12 patients were obese (BMI $>28$ ).

The most common histology of synchronous ovarian and endometrial tumour is endometrial type ${ }^{[7,13,16]}$ In the present study, $86 \%$ of endometrial cancers and $67 \%$ of ovarian cancers were endometrial. We experienced that only $17 \%$ of endometrial and $25 \%$ of ovarian were poorly differentiated which was concordant with a $30 \%$ incidence typically detected in Stage I ovarian cancer. The incidence of myometrium invasion was $50 \%$ and there was no incidence of vascular invasion.

According to literature, most patients with synchronous cancers present with symptoms characteristic of endometrial cancer. Abnormal uterine bleeding and abdominal pain were the main presenting symptoms.

In our series, median follow-up was 9 months (range: 4-41 months). Three patients experienced relapse. Other 
Table 3: Tumour characteristics ovarian cancer

\begin{tabular}{l|c}
\hline Characteristics & Frequency number (\%) \\
\hline Stage & $5(42)$ \\
\hline IA & $3(25)$ \\
\hline IB & $2(17)$ \\
\hline IC & $2(16)$ \\
\hline IIIA & \\
\hline Grade & $6(50)$ \\
\hline I & $2(17)$ \\
\hline II & $3(25)$ \\
\hline III & $1(8)$ \\
\hline Unknown & $8(67)$ \\
\hline Histology & $1(8)$ \\
\hline Endometrial & $1(8)$ \\
\hline Clear & $2(17)$ \\
\hline Serous & \\
\hline Mucinous & $10(84)$ \\
\hline Adjuvant chemotherapy & $2(16)$ \\
\hline Yes & \\
\hline No &
\end{tabular}

patients are alive without relapse. Given the small number of patients, no overall or disease-free survival data could be ascertained.

Our study was conducted in a single institution rather than multicentre analysis. The major limitation of the study was limited number and retrospective review. The diagnosis of synchronous malignancy was confirmed only after pathologic examinations, and it was hard to predict before surgical staging.

Guidelines for adjuvant treatment in patients with synchronous cancers have not been yet established. It is not possible to make definitive conclusion on optimal treatment. The excellent outcome in low-stage, low-grade tumours treated with surgery alone, however, supports the merits of this approach. Patients with more advanced stage, unfavourable histologies and high-grade disease should receive treatment tailored to both tumours.

\section{Conclusion}

The incidence of synchronous endometrial and ovarian cancer is remarkable, especially in premenopausal or young woman of reproductive age. Synchronous carcinomas show very favourable pathological features compared with the general endometrial or ovarian cancers. Treatment should be tailored to the histology, stage and grade of the individual tumours.

\section{Conflict of Interest}

The authors declare that they have no conflict of interest.

\section{References}

1. Ayhan A, Yalçin OT, Tuncer ZS, et al. Synchronous primary malignancies of the female genital tract. Eur J Obstet Gynecol Reprod Biol 1992;45:63-6.

2. Tong SY, Lee YS, Park JS, et al. Clinical analysis of synchronous primary neoplasms of the female reproductive tract. Eur J Obstet Gynecol Reprod Biol 2008;136:78-82.

3. Eisner RF, Nieberg RK, Berek JS. Synchronous primary neoplasms of the female reproductive tract. Gynecol Oncol 1989;33:335-9.

4. Chiang YC, Chen CA, Huang CY, et al. Synchronous primary cancers of the endometrium and ovary. Int $\mathrm{J}$ Gynecol Cancer 2008; 18:159-64.

5. Woodruff JD, Solomon D, Sullivant H. Multifocal disease in the upper genital canal. Obstet Gynecol 1985;65:695-8.

6. Lauchlan SC. The secondary müllerian system. Obstet Gynecol Surv 1972;27:133-46.

7. Soliman PT, Slomovitz BM, Broaddus RR, et al. Synchronous primary cancers of the endometrium and ovary: A single institution review of 84 cases. Gynecol Oncol 2004;94:456-62.

8. Scully RE, Young RH, Clement PB. Tumors of the Ovary, Maldeveloped Gonads, Fallopian Tube, and Broad Ligament. Atlas of Tumor Pathology. Vol. 189. Washington, DC: Armed Forces Institute of Pathology; 1998. p. 145.

9. Annegers JF, Malkasian GD Jr. Patterns of other neoplasia in patients with endometrial carcinoma. Cancer 1981;48:856-9.

10. Czernobilsky B, Silverman BD, Mikuta JJ. Endometrioid carcinoma of the ovary. Cancer 1970;26:1141-52.

11. Klemi KJ, Gronoos M. Endometrioid carcinoma of the ovary. A clinicopathological histochemical, and electronmicroscopic study. Obstet Gynecol 1979;53:572-9.

12. Kottmeier HL. The diagnosis and treatment of ovarian malignancies. Arq Patol 1965;37:51-64.

13. Walsh C, Holschneider C, Hoang Y, et al. Coexisting ovarian malignancy in young women with endometrial cancer. Obstet Gynecol 2005;106:693-9.

14. Herrinton LJ, Voigt LF, Weiss NS, et al. Risk factors for synchronous primary endometrial and ovarian cancers. Ann Epidemiol 2001;11:529-33.

15. Nishimura $\mathrm{N}$, Hachisuga $\mathrm{T}$, Yokoyama $\mathrm{M}$, et al. Clinicopathologic analysis of the prognostic factors in women with coexistence of endometrioid adenocarcinoma in the endometrium and ovary. J Obstet Gynaecol Res 2005;31:120-6.

16. Zaino R, Whitney C, Brady MF, et al. Simultaneously detected endometrial and ovarian carcinomas-a prospective clinicopathologic study of 74 cases: A gynecologic oncology group study. Gynecol Oncol 2001;83:355-62. 